\title{
Community Interventions
}

Lakshmi Vijayakumar

Dr. Lakshmi Vijayakumar is the Head of SNEHA Suicide prevention Centre in Chennai. She is particularly interested in the combination of clinical and public health interventions and suicide prevention possibilities within them. She has been conferred 'Honorary Fellowship' of the Royal College of Psychiatrists (FR(Psych), UK for her work on suicide prevention locally, nationally and globally. She is the first woman psychiatrist from South East Asia to receive this honour. Dr. Vijayakumar was a guest of the Triple i conference for the first time in 2014 when she spoke about the relevance of formulating locally relevant, culturally appropriate, costeffective and sustainable interventions.

Abstract. Suicide rates vary widely across countries and within regions in each country. Developing a 'one size fits all' suicide prevention programmes are unlikely to bring down the suicide rates. A bottoms up reach is also needed. It is crucial to develop cost effective and culturally appropriate interventions involving the community. The major challenges in developing such interventions are identifying and understanding the sociocultural context of the community, engaging with different sections of the community without compromising evidence base or effectiveness of suicide interventions, task shifting and ensuring sustainability. Examples of community suicide prevention interventions from high, middle and low-income countries and for vulnerable sections of the community will be discussed. 